\title{
ORIGINAL ARTICLE \\ Longitudinal association between lifestyle and coronary heart disease risk factors among individuals with spinal cord injury
}

\author{
S de Groot ${ }^{1,2}$, MW Post ${ }^{3}$, GJ Snoek ${ }^{4}$, M Schuitemaker ${ }^{4}$ and LH van der Woude ${ }^{2,5}$
}

Objective: To investigate: (1) the course of coronary heart disease risk factors (lipid profiles and body mass index (BMI)) in the first five years after discharge from inpatient spinal cord injury ( $\mathrm{SCl}$ ) rehabilitation and (2) the association between lifestyle (physical activity, self-care related to fitness, smoking, alcohol, body mass and low-fat diet) and coronary heart disease risk factors during that period.

Design: Prospective cohort study.

Participants/methods: Individuals with SCI $(N=130)$. Total cholesterol $(\mathrm{TC})$, high-density lipoprotein (HDL), low-density lipoprotein (LDL), triglycerides (TG) and BMI were determined at discharge from inpatient rehabilitation and 1 and 5 years after discharge. Using multilevel regression models, the effects of lifestyle (drinking alcohol, smoking, active lifestyle and self-care) on the lipid profiles and BMI were determined.

Results: After correction for lesion and personal characteristics, no changes in lipid profiles in the five years after discharge were seen, whereas the BMI increased significantly with $1.8 \mathrm{~kg} \mathrm{~m}^{-2}$. A high percentage was at risk of cardiovascular disease due to high BMI $(63-75 \%)$ or HDL (66-95\%). The individuals who indicated to maintain their fitness level as good as possible and the individuals with a low BMI showed better lipid profiles. Individuals with a more active lifestyle showed higher HDL levels. Individuals who avoid smoking showed a $1.5 \mathrm{~kg} \mathrm{~m}^{-2}$ higher BMI.

Conclusion: Lipid profiles seem to stabilize in the years after discharge from inpatient SCI rehabilitation, whereas the BMI increased. Lifestyle factors associated with a favorable lipid profile and BMI could be identified.

Spinal Cord (2013) 51, 314-318; doi:10.1038/sc.2012.153; published online 4 December 2012

Keywords: spinal cord injury; cardiovascular disease; lipid profile; body mass index; lifestyle

\section{INTRODUCTION}

Cardiovascular disease is one of the most common causes of death in adults with spinal cord injury (SCI). ${ }^{1}$ Dislipidemia $^{2}$ and obesity ${ }^{3}$ are more common among individuals with SCI compared with the general population, which leads to a higher risk of cardiovascular disease in the population with SCI. The course of the lipid profile over the five years after inpatient rehabilitation has not yet been studied. Although there was a positive change in lipid profiles during rehabilitation, the lipid profiles as well as the BMI showed unfavorable changes in the year after discharge from inpatient rehabilitation. ${ }^{4,5}$ These results showed the importance of a followup measurement on a longer term after discharge from rehabilitation.

Changes in body mass or lipid profile in people with SCI can be due to metabolic abnormalities or inactivity as a consequence of the paralysis. ${ }^{6}$ However, behavioral factors can also be associated with unfavorable changes in body mass and lipid profile. Previous studies found an effect of smoking, ${ }^{7}$ alcohol consumption, body mass, active lifestyle ${ }^{7}$ and fitness ${ }^{8}$ on lipid profiles in individuals with SCI. The effect of these lifestyle factors on body mass seems less clear ${ }^{7}$ or has not yet been investigated. Owing to their paralysis and subsequent sedentary lifestyle, a healthy lifestyle is particularly important for individuals with an SCI to keep a favorable BMI or lipid profile.

The purpose of this study is to investigate the association between lifestyle (physical activity, self-care related to fitness, smoking, alcohol, body mass and diet) and cardiovascular disease risk factors (lipid profiles and body mass index (BMI)) during the first five years after discharge from inpatient SCI rehabilitation.

\section{MATERIALS AND METHODS}

The current study was part of the Dutch prospective cohort study 'Physical strain, work capacity and mechanisms of restoration of mobility in the rehabilitation of individuals with SCI. ${ }^{\prime}$ Participants from eight rehabilitation centers that are specialized in SCI rehabilitation in the Netherlands were included between August 2000 and July 2003. Inclusion criteria were: acute SCI, between 18-65 years of age, classified as A-D on the American Spinal Injury Association (ASIA) impairment scale, (partly) wheelchair dependent, not having a progressive disease (for example, malignant tumor) or a psychiatric problem that interferes with constructive study participation according to the physician, and sufficient understanding of the Dutch language to understand the purpose of the study and the testing methods. One hundred and thirty individuals participated in the present study.

${ }^{1}$ Amsterdam Rehabilitation Research Center, Reade, Amsterdam, the Netherlands; ${ }^{2}$ Center for Human Movement Sciences, UMCG, University of Groningen, Groningen, The Netherlands: ${ }^{3}$ Rudolf Magnus Institute of Neuroscience and Center of Excellence in Rehabilitation Medicine, University Medical Center Utrecht and De Hoogstraat, Utrecht, The Netherlands; ${ }^{4}$ Roessingh Rehabilitation Center, Enschede, The Netherlands and ${ }^{5}$ Center for Rehabilitation, UMCG, University of Groningen, The Netherlands 
The Medical Ethics Committee of the Stichting Revalidatie Limburg/Institute for Rehabilitation Research in Hoensbroek approved the research protocol in 1999 and the Medical Ethics Committee of the University Hospital of Utrecht approved the follow-up research protocol in 2006. All participants completed an informed consent form.

\section{Design}

Data for the current study were collected at discharge from inpatient rehabilitation (T1), 1 year (T2) and 5 years (T3) after discharge from inpatient rehabilitation by trained research assistants with a paramedical background using standardized procedures.

\section{Blood lipids and BMI}

Blood samples were taken in the morning, when the individuals were in a fasting state. Total cholesterol (TC, in mmoll ${ }^{-1}$ ) and triglycerides (TG, in mmoll ${ }^{-1}$ ) concentrations were measured using standardized enzymatic procedures. Highdensity lipoproteins ( $\mathrm{HDL}$, in $\mathrm{mmoll}^{-1}$ ) was determined after selective precipitation of the very low-density lipoprotein and low-density lipoprotein fractions (LDL, in mmoll ${ }^{-1}$ ); LDL was calculated using the Friedewald equation. ${ }^{10}$ The criteria of a lipid profile indicating an increased risk of cardiovascular disease were: TG level $>2.15 \mathrm{mmoll}^{-1} ; ;^{7}$ TC level of $11.11 \mathrm{mmoll}^{-1}$ or more; ${ }^{11}$ LDL level $>5.56 \mathrm{mmoll}^{-1.11} \mathrm{HDL}$ level $\leqslant 2.22 \mathrm{mmoll}^{-1.11}$ and a TC/HDL ratio $>7.0 .^{12}$

The body mass of the participant was assessed at each test occasion. The height of the participant was asked at the start of inpatient rehabilitation. The BMI was calculated as body mass/height $\left(\mathrm{kg} \mathrm{m}^{-2}\right)$. Participants with a BMI $\geqslant 22 \mathrm{~kg} \mathrm{~m}^{-2}$ were classified as overweight/obese, ${ }^{13}$ following SCI-specific guidelines, and at risk of cardiovascular disease.

\section{Lifestyle factors}

Information on the level of physical activity (leisure, household and occupational activity) was collected using the physical activity scale for individuals with physical disabilities (PASIPD). ${ }^{14}$ The PASIPD consists of 13 questions, of which the first question is included to familiarize respondents with the item format and not scored, and question 10 on lawn work or yard care and question 11 on outdoor gardening were merged into a single question, since this better represented the Dutch situation. A total physical activity score is computed from the 11 remaining questions, expressed in metabolic equivalent (METs in hour day ${ }^{-1}$, with a maximum score of 182.3 MET hour day ${ }^{-1}$. One MET is defined as the amount of oxygen required per minute under quiet resting conditions.

The Health Behavior Scale ${ }^{15}$ was administered 1 year after discharge from inpatient rehabilitation and measures specific health behaviors needed for individuals living with SCI. It is a self-report questionnaire and consists of 22 descriptions of health behaviors. The present study used five questions of the Health Behavior Scale: (1) I maintain my physical fitness as good as possible; (2) I avoid smoking (of cigarettes, cigars and pipes); (3) I avoid alcohol use; (4) I make sure that my body mass does not increase and (5) I limit the amount of fat and cholesterol in my food (for example, I limit eating red meat and dairy products). Participants were asked to state the occurrence of the behaviors (never $=1$, sometimes $=2$, often $=3$ and always $=4$ ). The answers on these questions were dichotomized $(0=$ never or sometimes; $1=$ often or always).

\section{Personal and lesion characteristics}

Information about personal (age and sex) and lesion characteristics (level and completeness) were collected at discharge. Lesion characteristics were assessed according to the International Standards for Neurological Classification of Spinal Cord Injury. Neurological levels below T1 were defined as paraplegia, neurological levels at or above T1 were defined as tetraplegia. Participants were also divided into three subgroups based on sympathetic pathways to both the heart and vascular system: individuals with cervical lesions; lesions at the levels T1-T5 and lesions at T6 or below. AIS grades A and B were considered motor complete, and grades $\mathrm{C}$ and $\mathrm{D}$ were considered motor incomplete.

\section{Statistical analysis}

Descriptive statistics for personal and lesion characteristics, lipid profiles, BMI and lifestyle factors were calculated.

Changes in lipid profiles and BMI after discharge from inpatient rehabilitation were studied using multilevel regression analyses. This analysis accounts for the dependency and hierarchical nature of repeated measures. Furthermore, the number of observations per individual may vary, so that individuals with incomplete data can be included in the analyses. ${ }^{16}$ TC, TG, HDL, LDL, TC/ HDL and BMI were the dependent variables of the different regression analyses. All individuals with valid data on two or more test occasions were included in the analysis.

Firstly, each dependent variable was modeled over time since discharge, using time periods between the test occasions as categorical variables (dummy) with discharge from inpatient rehabilitation (T3) as reference. The regression coefficient for a time dummy describes the change in the dependent variable over that time period.

Secondly, all dependent variables were dichotomized into individuals having favorable or unfavorable lipid profiles or BMI, and six binomial random coefficient analyses were performed to investigate changes over time of the percentages of individuals at risk of unfavorable lipid profiles or BMI.

Thirdly, the longitudinal relationships between lesion, personal and lifestyle characteristics and the dichotomized variables of lipid profiles or BMI were investigated. Independent variables were lesion level (tetraplegia $=0$, paraplegia $=1$ ), motor completeness of the lesion (incomplete $=0$, complete $=1)$, age, sex $(\operatorname{men}=0$, women $=1)$, PASIPD score one year after discharge and self-care 1 year after discharge $(0=$ never/sometimes, $1=\mathrm{often} /$ always). BMI at every test occasion was also entered as an independent variable in the models with the lipid profiles as dependent variable. Furthermore, the interactions between the independent variables and time were entered in the regression analyses to investigate possible differences in course over time

Table 1 Descriptives for lipid profile and BMI at discharge from inpatient rehabilitation and 1 and 5 years later

Model data (Individuals with $\geqslant 2$ measurements)

\begin{tabular}{|c|c|c|c|c|c|c|c|c|c|}
\hline & \multicolumn{3}{|c|}{ Discharge } & \multicolumn{3}{|c|}{1 year after discharge } & \multicolumn{3}{|c|}{5 years after discharge } \\
\hline & $N$ & Mean (s.d.) & $\%$ At risk & $N$ & Mean (s.d.) & $\%$ At risk & $N$ & Mean (s.d.) & \%At risk \\
\hline BMI $\left(\mathrm{kg} / \mathrm{m}^{2}\right)$ & 125 & $23.7(4.1)$ & 63 & 116 & $24.4(4.6)$ & 68 & 97 & $25.5(4.7)$ & 74 \\
\hline $\mathrm{TC}(\mathrm{mmol} / \mathrm{l})$ & 126 & $4.73(1.02)$ & 3 & 108 & 4.98 (1.17) & 10 & 74 & $4.83(1.02)$ & 32 \\
\hline $\mathrm{HDL}(\mathrm{mmol} / \mathrm{l})$ & 124 & $1.17(0.37)$ & 95 & 109 & $1.22(0.40)$ & 88 & 74 & $1.24(0.44)$ & 66 \\
\hline LDL (mmol/l) & 122 & $2.90(0.87)$ & 6 & 106 & $3.12(0.99)$ & 13 & 70 & $2.96(0.86)$ & 35 \\
\hline $\mathrm{TG}(\mathrm{mmol} / \mathrm{l})$ & 126 & $1.52(0.91)$ & 13 & 108 & $1.57(1.07)$ & 16 & 73 & $1.62(1.38)$ & 16 \\
\hline $\mathrm{TC} / \mathrm{HDL}$ & 124 & $4.43(1.86)$ & 6 & 108 & $4.46(1.72)$ & 10 & 74 & $4.25(1.43)$ & 5 \\
\hline
\end{tabular}

Abbreviations: BMI, body mass index; HDL, high-density lipoprotein; LDL, low-density lipoprotein; \% at risk, Percentage at risk of cardiovascular disease when using the cut-off values described in the methods; \% at risk ATP III, Percentage at risk of cardiovascular disease when using the guidelines of the adult team panel (ATP) III guidelines of the National Cholesterol Education Program; s.d., standard deviation; TC, Total cholesterol; TG, triglycerides. 
between subgroups. All independent variables were added in separate analyses to the basic model with the time dummies. Independent variables with $P$-values $<0.1$ in this model were included in a subsequent multivariate regression model. Using the backward selection procedure, stepwise excluding nonsignificant determinants $(P>0.05)$ and final multivariate models for each dependent variable were created.

The longitudinal relationships were also performed with three lesion groups (inserting two dummies; in one model, tetraplegia was the reference and in the other, low paraplegia).

\section{RESULTS}

\section{Descriptives}

One hundred and thirty individuals participated in two or more test occasions. A total of 225 individuals with SCI entered the study at the start of inpatient rehabilitation. Reasons for 95 dropouts between the first and the last measurement of the research program were that individuals died, refused to collaborate, moved, could not be contacted or had other reasons to decline participation. The average age of our sample was $40.1 \pm 13.8$ years. In all, $70 \%$ were men, $34 \%$ had a tetraplegia and $35 \%$ had a motor incomplete lesion. At the start of rehabilitation, participants did not significantly differ from dropouts regarding gender $(P=0.06$; dropouts: $81 \%$ men), age $(P=0.19$; dropouts: $42.1 \pm 14.5$ years), lesion level ( $P=0.08$; dropouts: $47 \%$ tetraplegia), completeness $(P=0.38)$, lipid profile $(P=0.35-0.86)$ and BMI $(P=0.62)$.

The average PASIPD score 1 year after discharge was 17.7 (s.d. = 18.3) MET h day $^{-1}$. Table 1 shows the descriptive results of the BMI and lipid profile. Figure 1 shows the answers on the self-care questions 1 year after discharge. The majority of the individuals with SCI were at risk of being overweight/obese (63-74\%) or having a low HDL level (66-95\%). The percentage at risk of other unfavorable lipid levels was clearly lower (3-35\%) (Table 1 ). Only $0.8-4.4 \%$ of the group was not at risk of TC, HDL, LDL, TG or BMI on the three test occasions, whereas others were at risk of one (25-35\%), two

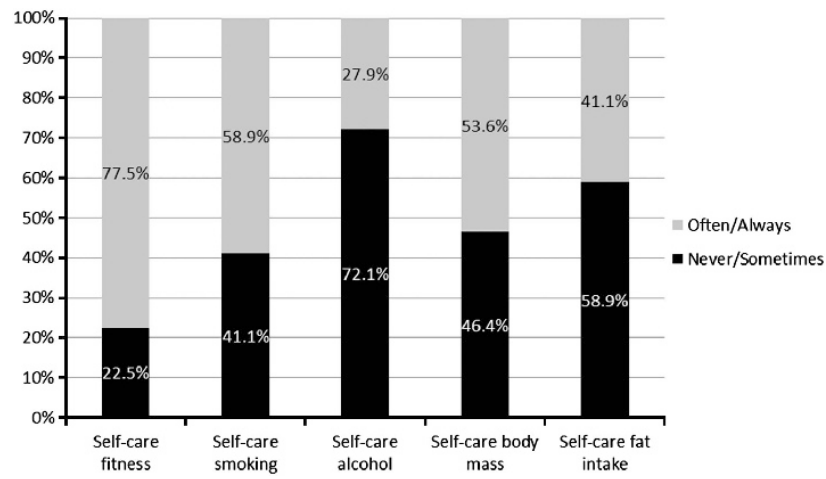

Figure 1 Answers on the self-care questions at 1 year after discharge.
(52-57\%), three $(10-12 \%)$ or four $(1.6-2.9 \%)$ of these factors for cardiovascular disease.

\section{Course}

No significant changes in lipid profiles were found after discharge from inpatient rehabilitation (Table 2), whereas the BMI increased significantly with $1.8 \mathrm{~kg} \mathrm{~m}^{-2}$ between discharge and 5 years later (Table 2). Extra analyses including only the participants that performed all three measurements showed similar results.

The percentage of participants at risk of cardiovascular disease based on the cutoff points for TC, HDL and LDL changed significantly over time (Table 3). Compared with the time of discharge, 5 years later, the participants were at a higer risk of cardiovascular disease based on TC (14 times more risk) and LDL (8.3 times more risk), and were 9.2 times less at risk based on HDL.

\section{Personal, lesion and lifestyle characteristics}

Table 4 shows the associations between lipid profiles and BMI with the personal, lesion and lifestyle characteristics. Age was significantly unfavorably related to TC, LDL, TG and BMI. Women had significantly higher HDL levels and a significantly lower TC/HDL ratio. Individuals with a complete lesion had significantly lower HDL levels and those with paraplegia had significantly higher TG levels. The analysis with three lesion groups showed only a difference in LDL : the group with a high paraplegia differed from the groups with a tetraplegia and low paraplegia.

The self-care question 'I maintain my physical fitness as good as possible' related to a favorable lipid profile (significantly higher HDL and lower TC, LDL and TG). Those individuals with an active lifestyle according to the PASIPD had more favorable HDL levels. A higher BMI led to unfavorable lipid profiles, that is, significantly lower HDL and higher TC, LDL and TG levels. Individuals who often or always avoid smoking had a $1.5 \mathrm{~kg} \mathrm{~m}^{-2}$ higher BMI than individuals who never or sometimes avoid smoking.

No interaction effects were found between time and any of the personal, lesion or lifestyle characteristics.

\section{DISCUSSION}

Although the BMI increased significantly during the 5 years after discharge and was related to all lipid profiles, the lipid profiles remained stable during the 5 years after discharge. As far as known, only two studies have looked at the course of lipid profiles after SCI but on a shorter term, that is during about 2 years post injury. ${ }^{4,17}$ During these 2 years, post-injury changes in lipid profiles were seen. Although a significant increase was found in LDL and TC in the first year after discharge, ${ }^{4}$ these values showed a (non-signifcant) decrease between the 1st and 5th year after discharge. Although the BMI increased significantly after discharge and was significantly related to all lipid profiles, the increase in BMI was not accompanied by a

Table 2 The basic multilevel regression model (time dummies included only) for the lipid profiles and BMI

\begin{tabular}{|c|c|c|c|c|c|c|c|c|c|c|c|c|}
\hline & \multicolumn{2}{|c|}{ Total cholesterol } & \multicolumn{2}{|l|}{$H D L$} & \multicolumn{2}{|l|}{$L D L$} & \multicolumn{2}{|l|}{ Triglycerides } & \multicolumn{2}{|l|}{$T C / H D L$} & \multicolumn{2}{|c|}{ Body mass index } \\
\hline & Beta (s.e.) & $\mathrm{P}$ & Beta (s.e.) & $\mathrm{P}$ & Beta (s.e.) & $P$ & Beta (s.e.) & $\mathrm{P}$ & Beta (s.e.) & $\mathrm{P}$ & Beta (s.e.) & $\mathrm{P}$ \\
\hline Constant & $4.833(0.124)$ & & $1.241(0.046)$ & & $2.956(0.109)$ & & $1.605(0.188)$ & & $4.254(0.199)$ & & $25.493(0.450)$ & \\
\hline Discharge-5 yrs & $-0.104(0.156)$ & 0.51 & $-0.075(0.058)$ & 0.20 & $-0.058(0.136)$ & 0.67 & $-0.096(0.180)$ & 0.60 & $0.179(0.251)$ & 0.48 & $-1.762(0.599)$ & 0.003 \\
\hline $1 \mathrm{yr}-5 \mathrm{yrs}$ & $0.143(0.161)$ & 0.38 & $-0.018(0.060)$ & 0.76 & $0.165(0.140)$ & 0.24 & $-0.045(0.190)$ & 0.81 & $0.208(0.258)$ & 0.42 & $-1.046(0.609)$ & 0.09 \\
\hline
\end{tabular}

Abbreviations: HDL, high-density lipoprotein; LDL, low-density lipoprotein; TC, Total cholesterol. 
Table 3 The basic multilevel binomial regression model (time dummies included only) to investigate the change over time for percentage of participants at risk $(=1)$ vs not at risk $(=0)$ for unfavorable lipid profiles or BMI

\begin{tabular}{|c|c|c|c|c|c|c|c|}
\hline & \multirow{2}{*}{$\begin{array}{l}\text { Constant } \\
\text { Beta (s.e.) }\end{array}$} & \multicolumn{3}{|c|}{ Discharge-5 yrs } & \multicolumn{3}{|c|}{$1 y r-5 y r s$} \\
\hline & & Beta (s.e.) & Odds Ratio & P-value & Beta (s.e.) & Odds Ratio & P-value \\
\hline Total cholesterol & $-0.778(0.207)$ & $-2.672(0.546)$ & 0.07 & $<0.001$ & $-1.420(0.368)$ & 0.24 & $<0.001$ \\
\hline HDL & $0.652(0.203)$ & $2.214(0.435)$ & 9.20 & $<0.001$ & $1.294(0.342)$ & 3.65 & $<0.001$ \\
\hline LDL & $-0.638(0.203)$ & $-2.087(0.418)$ & 0.12 & $<0.001$ & $-1.308(0.343)$ & 0.27 & $<0.001$ \\
\hline Triglycerides & $-1.626(0.316)$ & $-0.302(0.414)$ & 0.74 & 0.94 & $0.685(0.604)$ & 1.98 & 0.26 \\
\hline $\mathrm{TC} / \mathrm{HDL}$ & $-2.862(0.514)$ & $0.046(0.644)$ & 1.05 & 0.94 & $0.685(0.604)$ & 1.98 & 0.26 \\
\hline Body mass index & $1.058(0.232)$ & $-0.517(0.297)$ & 0.60 & 0.08 & $-0.299(0.306)$ & 0.74 & 0.33 \\
\hline
\end{tabular}

Abbreviations: HDL, high-density lipoprotein; LDL, low-density lipoprotein; TC, Total cholesterol.

Table 4 Final multivariate regression models for the different lipid profiles and BMI

\begin{tabular}{|c|c|c|c|c|c|c|c|c|c|c|c|c|}
\hline & \multicolumn{2}{|c|}{$T C$} & \multicolumn{2}{|c|}{$H D L$} & \multicolumn{2}{|l|}{$L D L$} & \multicolumn{2}{|c|}{$T G$} & \multicolumn{2}{|c|}{$T C / H D L$} & \multicolumn{2}{|c|}{ Body mass index } \\
\hline & Beta (s.e.) & $P$ & Beta (s.e.) & $P$ & Beta (s.e.) & $P$ & Beta (s.e.) & $P$ & Beta (s.e.) & $\mathrm{P}$ & Beta (s.e.) & $P$ \\
\hline Constant & $\begin{array}{l}3.289 \\
(0.395)\end{array}$ & & $\begin{array}{l}1.639 \\
(0.160)\end{array}$ & & $\begin{array}{c}1.784 \\
(0.345)\end{array}$ & & $\begin{array}{l}-0.855 \\
(0.421)\end{array}$ & & $\begin{array}{l}1.976 \\
(0.677)\end{array}$ & & $\begin{array}{l}21.139 \\
(0.847)\end{array}$ & \\
\hline Discharge-5 yrs & $\begin{array}{c}0.072 \\
(0.163)\end{array}$ & 0.66 & $\begin{array}{l}-0.122 \\
(0.058)\end{array}$ & 0.04 & $\begin{array}{c}0.042 \\
(0.143)\end{array}$ & 0.77 & $\begin{array}{c}0.138 \\
(0.192)\end{array}$ & 0.47 & $\begin{array}{c}0.519 \\
(0.252)\end{array}$ & 0.04 & $\begin{array}{l}-1.867 \\
(0.602)\end{array}$ & 0.002 \\
\hline $1-5$ years & $\begin{array}{c}0.253 \\
(0.164)\end{array}$ & 0.12 & $\begin{array}{l}-0.043 \\
(0.058)\end{array}$ & 0.46 & $\begin{array}{c}0.240 \\
(0.143)\end{array}$ & 0.09 & $\begin{array}{c}0.050 \\
(0.195)\end{array}$ & 0.80 & $\begin{array}{c}0.377 \\
(0.252)\end{array}$ & 0.14 & $\begin{array}{l}-1.128 \\
(0.603)\end{array}$ & 0.06 \\
\hline Age & $\begin{array}{c}0.019 \\
(0.005)\end{array}$ & $<0.001$ & NS & - & $\begin{array}{c}0.009 \\
(0.004)\end{array}$ & 0.02 & $\begin{array}{c}0.009 \\
(0.004)\end{array}$ & 0.02 & NS & - & $\begin{array}{c}0.086 \\
(0.017)\end{array}$ & $<0.001$ \\
\hline $\operatorname{Sex}^{a}$ & NS & - & $\begin{array}{c}0.215 \\
(0.047)\end{array}$ & $<0.001$ & NS & - & NS & - & $\begin{array}{l}-0.540 \\
(0.207)\end{array}$ & 0.009 & NS & - \\
\hline Completeness $^{b}$ & NS & - & $\begin{array}{l}-0.123 \\
(0.046)\end{array}$ & 0.008 & NS & - & NS & - & NS & - & NS & - \\
\hline Lesion level ${ }^{c}$ & NS & - & NS & - & NS & - & $\begin{array}{l}0.322 \\
(0.130)\end{array}$ & 0.01 & NS & - & NS & - \\
\hline $\begin{array}{l}\text { Self-care fitness at } 1 \\
\text { year }^{d}\end{array}$ & $\begin{array}{l}-0.420 \\
(0.148)\end{array}$ & 0.005 & $\begin{array}{c}0.141 \\
(0.053)\end{array}$ & 0.008 & $\begin{array}{l}-0.367 \\
(0.130)\end{array}$ & 0.005 & $\begin{array}{l}-0.473 \\
(0.144)\end{array}$ & 0.001 & $\begin{array}{l}-1.196 \\
(0.231)\end{array}$ & $<0.001$ & NS & - \\
\hline $\begin{array}{l}\text { Self-care smoking at } \\
1 \text { yeard }^{d}\end{array}$ & NS & - & NS & - & NS & - & NS & - & NS & - & $\begin{array}{l}1.517 \\
(0.489)\end{array}$ & 0.002 \\
\hline $\begin{array}{l}\text { Self-care alcohol at } \\
1 \text { yeard }^{d}\end{array}$ & NS & - & NS & - & NS & - & NS & - & NS & - & NS & - \\
\hline $\begin{array}{l}\text { Self-care body mass } \\
\text { at } 1 \text { yeard }\end{array}$ & NS & - & NS & - & NS & - & NS & - & NS & - & NS & - \\
\hline $\begin{array}{l}\text { Self-care fat intake } \\
\text { at } 1 \text { year }\end{array}$ & NS & - & NS & - & NS & - & NS & - & NS & - & NS & - \\
\hline PASIPD at 1 year & NS & - & $\begin{array}{l}0.003 \\
(0.001)\end{array}$ & 0.003 & NS & - & NS & - & NS & - & NS & - \\
\hline BMI & $\begin{array}{c}0.037 \\
(0.015)\end{array}$ & 0.01 & $\begin{array}{l}-0.030 \\
(0.005)\end{array}$ & $<0.001$ & $\begin{array}{c}0.039 \\
(0.013)\end{array}$ & 0.003 & $\begin{array}{l}0.086 \\
(0.014)\end{array}$ & $<0.001$ & $\begin{array}{c}0.151 \\
(0.022)\end{array}$ & $<0.001$ & - & - \\
\hline
\end{tabular}

Abbreviations: HDL, high-density lipoprotein; LDL, low-density lipoprotein; TC, Total cholesterol; TG, triglycerides. Note: All results are regression coefficients $(\beta)$ and standard error (s.e.) for the model after backward elimination. The regression coefficients represent the change in outcome associated with an

NS: Independent variable proven not significant (NS) in previous analyses and therefore not entered into this model.

a Men=0; women $=1$.

Incomplete=0; complete $=1$.

${ }^{\mathrm{C}}$ Tetraplegia $=0$; paraplegia $=1$.

${ }^{\mathrm{d}}$ Never/sometimes $=0 ;$ often/always $=1$

significant change in lipid profile over the years. The explained variance of the models with the lipid profiles as outcome measures and $\mathrm{BMI}$ as an independent variable was between $15-25 \%$, indicating that there are other factors that have a role besides BMI.

Our finding that a high percentage is at risk of cardiovascular disease due to a high BMI (63-74\%) was similar to what was found in the Stockholm SCI study, ${ }^{18}$ that is, $82-85 \%$ (also using the cutoff point of $>22 \mathrm{~kg} \mathrm{~m}^{-2}$ for individuals with a SCI). Therefore, BMI should be one of the first targets of interventions to prevent cardiovascular disease. However, not much research has been done in this field. Therefore, future studies should focus on the energy balance of individuals with SCI and should investigate what the best weight loss program is regarding diet and exercise. Furthermore, individuals with SCI should be educated early about being at a higher risk of obesity.

Moreover, a very high percentage at risk of cardiovascular disease was found in HDL (66-95\%), although this percentage at risk diminished during the 5 years after discharge from inpatient rehabilitation. This result was in contrast to the percentage at risk of cardiovascular disease based on TC and LDL; these percentages increased during those 5 years. The percentages are much higher than when using the older cutoff values, used in our previous study, ${ }^{4}$ for determining the risk of cardiovascular disease (for example, 17-22\% of our present group would be at risk of HDL when using the old 
cutoff values). When analyzing these kind of data, it is important to look at the cutoff values that were applied.

The results of the association between personal characteristics and lipid profiles and BMI were quite similar to our previous studies focusing on these relationships during inpatient rehabilitation. ${ }^{4,5}$ Moreover, in our previous paper, ${ }^{4}$ the BMI was negatively related to all lipid levels and the PASIPD was positively related to the HDL level.

Questions regarding self-care have not yet been associated to cardiovascular risk factors. The self-care question related to maintaining physical fitness was associated with all lipid profiles. Individuals who indicated that they maintain their fitness level showed a more favorable lipid profile. The association between actually measured physical fitness and lipid profile was previously demonstrated. Higher peak power output, peak oxygen uptake and muscle strength were found to be associated with favorable HDL and TG levels and subsequently a better TC/HDL ratio. ${ }^{19}$ It is interesting to see that a simple question about maintaining physical fitness is also associated strongly with the lipid profile.

Avoidance of smoking, led to a $1.5 \mathrm{~kg} \mathrm{~m}^{-2}$ higher BMI. In the general population, it was also found that smokers had a lower BMI than non-smokers. ${ }^{20}$ It was suggested that negative effects of smoking on food intake, such as anorexia and a reduced sense of smell and taste, may contribute to this inverse association. ${ }^{20}$ Although smoking seemed to have a positive effect on BMI, smoking should not be stimulated because of the known negative effect on other health issues such as lung cancer.

Surprisingly, the self-care questions regarding diet and body mass were not significantly associated with the BMI. A lower BMI was not associated to less fat and cholesterol intake or awareness about an increase in body mass. On the other hand, a previous study found that health behavior was significantly more applied in individuals who had a particular secondary complication. ${ }^{15}$ However, we did not find that individuals with a high BMI are more aware of the need to limit their fat intake or make sure that their body mass does not increase.

A limitation of this study is the loss to follow up at 5 years after discharge measurement. However, when performing the analyses with only those participants who performed all three measurements, the same course of lipid profiles and BMI were found. Although a relatively high percentage of individuals with a motor complete lesion was included, the results can be generalized to all wheelchair users with SCI.

\section{CONCLUSION}

The BMI increased significantly whereas the lipid profiles remained stable during the 5 years after inpatient rehabilitation. Lifestyle factors associated with a favorable lipid profile (self-care related to fitness level, active lifestyle and body mass) and BMI (self-care related to smoking) could be identified.

\section{DATA ARCHIVING}

There were no data to deposit.

\section{CONFLICT OF INTEREST}

The authors declare no conflict of interest.

\section{ACKNOWLEDGEMENTS}

We would like to thank all the participants, the eight participating rehabilitation centers and especially the research assistants for collecting all the data: Sacha van Langeveld (De Hoogstraat, Utrecht), Annelieke Niezen/Peter Luthart (Rehabilitation Center Amsterdam), Marijke Schuitemaker (Het Roessingh, Enschede), Karin Postma/Rogier Broeksteeg/Jan Kamberg (Rijndam Revalidatiecentrum, Rotterdam), Jos Bloemen (Hoensbroeck

Revalidatiecentrum, Hoensbroek), Hennie Rijken (Sint Maartenskliniek,

Nijmegen), Ferry Woldring (Beatrixoord, Haren) and Linda Valent

(Heliomare, Wijk aan Zee). ZON-Mw Rehabilitation program, grant no. 1435.0003 and no. 1435.0025 .

1 Garshick E, Kelley A, Cohen SA, Garrison A, Tun CG, Gagnon D et al. A prospective assessment of mortality in chronic spinal cord injury. Spinal Cord 2005; 43: 408-416.

2 Bauman WA, Adkins RH, Spungen AM, Maloney P, Gambino R, Waters RL. Ethnicity effect on the serum lipid profile in persons with spinal cord injury. Arch Phys Med Rehabil 1998; 79: 176-180.

3 Buchholz AC, Bugaresti JM. A review of body mass index and waist circumference as markers of obesity and coronary heart disease risk in persons with chronic spinal cord injury. Spinal Cord 2005; 43: 513-518.

4 de Groot S, Dallmeijer AJ, Post MW, Angenot EL, van den Berg-Emons RJ, Van Der Woude LH. Prospective analysis of lipid profiles in persons with a spinal cord injury during and 1 year after inpatient rehabilitation. Arch Phys Med Rehabil 2008; 89: 531-537.

5 de Groot S, Post MW, Postma K, Sluis TA, Van Der Woude LH. Prospective analysis of body mass index during and up to 5 years after discharge from inpatient spinal cord injury rehabilitation. J Rehabil Med 2010; 42: 922-928.

6 Apstein MD, George BC. Serum lipids during the first year following acute spinal cord injury. Metabolism 1998; 47: 367-370.

7 Janssen TW, van Oers CA, van Kamp GJ, TenVoorde BJ, van der Woude LH, Hollander AP. Coronary heart disease risk indicators, aerobic power, and physical activity in men with spinal cord injuries. Arch Phys Med Rehabil 1997; 78: 697-705.

8 Bostom AG, Toner MM, McArdle WD, Montelione T, Brown CD, Stein RA. Lipid and lipoprotein profiles relate to peak aerobic power in spinal cord injured men. Med Sci Sports Exerc 1991; 23: 409-414.

9 de Groot S, Dallmeijer AJ, Post MW, van Asbeck FW, Nene AV, Angenot EL et al. Demographics of the Dutch multicenter prospective cohort study 'Restoration of mobility in spinal cord injury rehabilitation'. Spinal Cord 2006; 44 668-675.

10 Friedewald WT, Levy RI, Fredrickson DS. Estimation of the concentration of low-density lipoprotein cholesterol in plasma, without use of the preparative ultracentrifuge. Clin Chem 1972; 18: 499-502.

11 U.S. Department of Health and Human Services. ATP III Guidelines At-a-glance. Quick Desk Reference Report No.: NIH Publication No. 01-3305, 2001.

12 Kromhout D, Obermann-De Boer G, Blokstra A, Verschuren W. Monitoring Cardiovas cular Diseases 1990. RIVM: Bilthoven, 1991, Report No.: 528901003.

13 Laughton GE, Buchholz AC, Martin Ginis KA, Goy RE. Lowering body mass index cutoffs better identifies obese persons with spinal cord injury. Spinal Cord 2009; 47 757-762.

14 Washburn RA, Zhu W, McAuley E, Frogley M, Figoni SF. The physical activity scale for individuals with physical disabilities: development and evaluation. Arch Phys Med Rehabil 2002; 83: 193-200.

15 Bloemen-Vrencken JH, De Witte LP, Post MW, van den Heuvel WJ. Health behaviour of persons with spinal cord injury. Spinal Cord 2007; 45: 243-249.

16 Twisk J. Applied Longitudinal Data Analysis for Epidemiology. A Practical Guide. Cambridge University Press: Cambridge, England, 2003.

17 Dallmeijer AJ, van der Woude LH, van Kamp GJ, Hollander AP. Changes in lipid, lipoprotein and apolipoprotein profiles in persons with spinal cord injuries during the first 2 years post-injury. Spinal Cord 1999; 37: 96-102.

18 Wahman K, Nash MS, Lewis JE, Seiger A, Levi R. Cardiovascular disease risk and the need for prevention after paraplegia determined by conventional multifactorial risk models: the Stockholm spinal cord injury study. J Rehabil Med 2011; 43: 237-242.

19 de Groot S, Dallmeijer AJ, Post MW, Angenot EL, Van Der Woude LH. The longitudinal relationship between lipid profile and physical capacity in persons with a recent spinal cord injury. Spinal Cord 2008; 46: 344-351.

20 French S, Jeffrey R. Weight concerns and smoking: a literature review. Ann Behav Med 1995; 17: 234-244. 